\title{
Colour Simplex coding for Brillouin distributed sensors
}

\author{
Sébastien Le Floch*a, Florian Sauser ${ }^{\mathrm{a}}$, Miguel Llera ${ }^{\mathrm{a}}$, Marcelo A. Soto ${ }^{\mathrm{b}}$, Luc Thévenaz ${ }^{\mathrm{b}}$ \\ ${ }^{a}$ HE-ARC, Rue de la Serre 7, CH-2610 Saint Imier, Switzerland; \\ ${ }^{b}$ EPFL, Swiss Federal Institute of Technology, Institute of Electrical Engineering, \\ SCI-STI-LT Station 11, CH-1015 Lausanne, Switzerland \\ *sebastien.lefloch@he-arc.ch; phone +41 3293022 49; fax +41 3293022 04; www.he-arc.ch
}

\begin{abstract}
The possibility to customize Simplex coding for long range Brillouin Optical Time Domain Analysis is demonstrated by "colouring" the sequences in the frequency domain. The coding gain is identical to the traditional intensity-modulated Simplex code, though with much simplified series of sequences. The frequency-hopping pulses in return-to-zero intensity-modulated format are generated with a Direct-Digital Synthesizer. The proof-of-concept is experimentally demonstrated with measurements over a $50 \mathrm{~km}$ range (100 km fibre-loop) and a 2 meter spatial resolution.
\end{abstract}

Keywords: Stimulated Brillouin scattering, fibre optics sensors, Simplex coding, distributed fibre sensors.

\section{INTRODUCTION}

Stimulated Brillouin scattering (SBS) has been widely exploited for distributed fibre sensing, since it is sensitive to strain and temperature. Brillouin Optical Time Domain Analysers (BOTDA) quantify the local SBS interaction between a pump pulse and a continuous probe wave counter-propagating along the fibre, through the intercession of a resonant acoustic wave $\mathrm{e}^{1,2}$. This way, it is possible to evaluate the local strain or temperature over fibre lengths of tens of kilometres and with meter spatial resolution ${ }^{3}$. However, practical limits in term of signal-to-noise ratio (SNR) are observed in BOTDA systems, since the power of the interacting waves cannot be increased endlessly: the probe power cannot exceed a given maximum value to limit pump depletion that would lead to systematic errors on local strain or temperature measurement ${ }^{4}$. The pump power is in turn practically limited by other depletion effects resulting from modulation instability and Raman scattering ${ }^{5}$.

Pulse coding techniques, increasing the global pump energy without raising the peak power and without compromising spatial resolution, have been intensively used during these past three years to extend the distance range of BOTDA systems. Unipolar (on/off) codes, such as Simplex, were first employed with meter spatial resolution for a distance range of $50 \mathrm{~km}^{6,7}$. Recently, bipolar (plus/minus i.e. gain/loss) coding using Golay codes has shown a potential maximum range of $100 \mathrm{~km}$, with an inherent robustness to pump depletion due to the compensation between gain and loss processes $^{8}$. Simultaneously, a novel kind of coding has been proposed, based on frequency-agile pseudo-random pulses, showing an additional $\sqrt{ } 2$ gain in SNR with respect to unipolar codes ${ }^{9}$. This coding technique however suffers from huge data processing, due to the large number of sequences required to properly retrieve all information in time and frequency. In this paper, a simplified frequency coding is presented, based on a customization of Simplex codes. Each pump pulse in a coded sequence has a distinct frequency generated by a Direct-Digital Synthesizer (DDS) in the microwave domain, but unlike the time/frequency coding ${ }^{9}$, the position of each frequency in the pulse sequence is fixed. The coding principle is presented and validated by performing distributed measurements over $50 \mathrm{~km}$ with a spatial resolution of 2 meter.

\section{COLOUR SIMPLEX CODE}

So far Simplex coding for BOTDA is based on on/off intensity modulation of pump pulses ${ }^{6,7,10}$, all pulses having the same frequency. The distributed Brillouin gain spectrum (BGS) is then simply reconstructed by step-wise incrementing the frequency difference between pump and probe waves. A drawback of this technique is the absolute requirement to repetitively launch all sequences in the Simplex codes at each frequency increment. For example, a code length of 127 requires at each frequency step 127 sequences to retrieve the local Brillouin information along the fibre. Even for the unipolar Golay code, 4 sequences are required for each frequency step ${ }^{8}$.

Fifth European Workshop on Optical Fibre Sensors, edited by Leszek R. Jaroszewicz, Proc. of SPIE Vol. 8794, 879437 - (C) 2013 SPIE · CCC code: 0277-786X/13/\$18 - doi: 10.1117/12.2025795 
The here presented novel coding, designated as Colour Simplex, originates from Simplex codes, on which a frequency coding of the pump pulses is superimposed, as shown in Figure 1: the code length is equal to the number of discrete frequencies scanning the BGS, each column of the matrix corresponding to a specific pump pulse frequency. For instance, as shown in the first matrix line in Figure 1, sequentially a pump pulse at frequency $f_{l}$ is launched, then no light in time bin 2 , then a pump pulse at frequency $f_{3}$, then no light in bin 4 , then a pump pulse at frequency $f_{5}$, then no light in bin 6 , and finally a pump pulse at frequency $f_{7}$. For a long enough code, it is therefore possible to reconstruct the distributed BGS along the fibre. Actually the number of sequences must match the number of scanned discrete frequencies and must be chosen equal to $2^{n}-1$ ( $n$ integer): to the best of our knowledge, this is the most condensed coding process in term of sequences. The number of sequences is comparable to what is required for classical BOTDA systems, but with the bonus of the provided coding gain, defined as the net SNR enhancement ${ }^{6,7}$. The BGS reconstruction is then simply realized by applying the pre-determined inverse matrix $\mathrm{S}^{-1}$ to the acquired data.

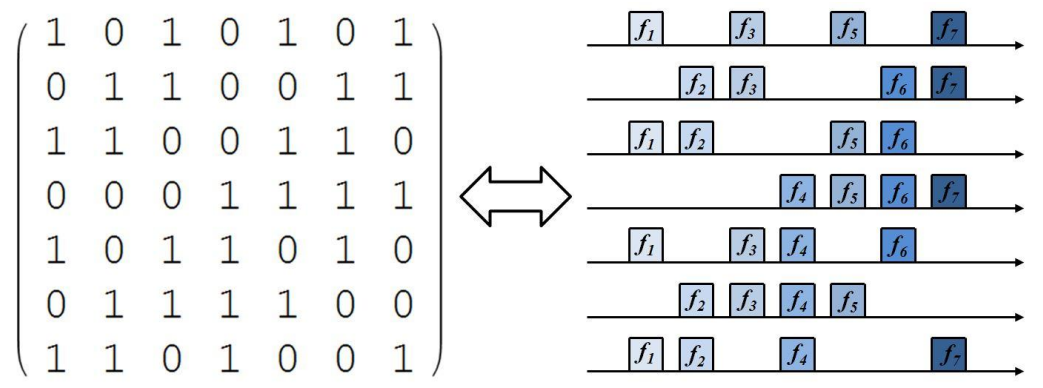

Figure 1: (left) Generating matrix $S$ of the Colour Simplex with a code length $L=7$,

(right) the corresponding sequences of pump pulses ( $f_{i}$ : discrete scanning pump frequencies).

\section{EXPERIMENTAL SET UP}

Figure 2 depicts the experimental set-up to validate Colour Simplex measurements: the output of a distributed feedback (DFB) laser source, operating at $1533 \mathrm{~nm}$, is split in distinct arms by an optical coupler. The continuous probe wave, directly tapped from the laser source, propagates in the lower arm through the first $50 \mathrm{~km}$ of fibre conveying the signal to the most distant end of the sensor, then through the $50 \mathrm{~km}$ sensing fibre and is detected after a circulator and a Bragg grating (FBG-1), this latter filtering out the coherent Rayleigh noise coming from the pump pulses. In the upper arm, an intensity modulator, operating in suppressed carrier mode, is driven by a DS frequency-agile microwave generator: the sequences of microwave frequencies generating the Colour Simplex code are loaded into the DDS prior to the acquisition. These frequencies are then doubled at the DDS output and fed into the intensity modulator. The lower sideband at the output of the IM is filtered out using a second Bragg grating (FBG-2). A polarization scrambler is used to mitigate the polarization-dependent Brillouin response along the fibre. The remaining sideband is then amplified using an erbium-doped fibre amplifier (EDFA) and the pump sequence power is monitored on a second detector after a coupler.

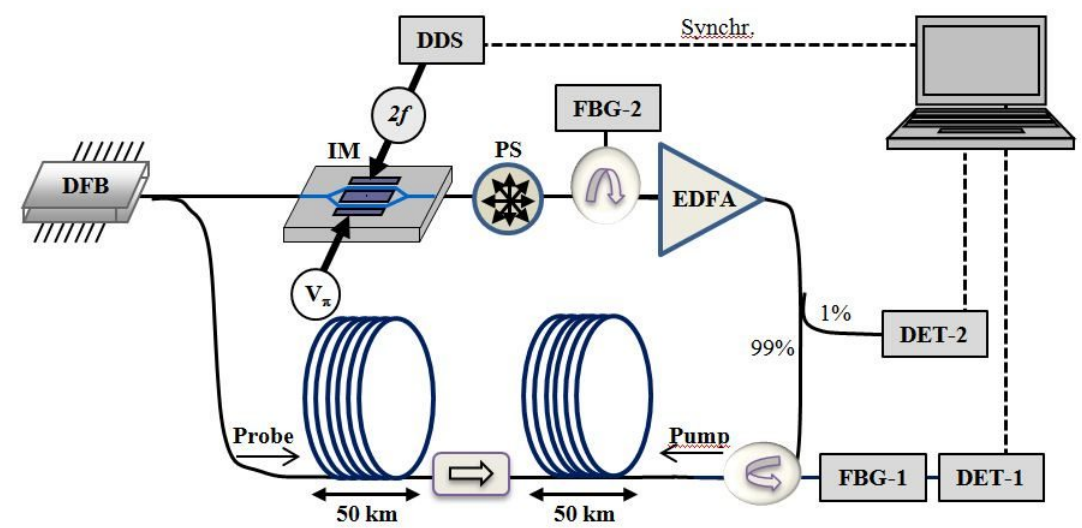

Figure 2: Experimental set-up. DDS: Direct-Digital Synthesizer; FBG: Fibre Bragg Grating; DET: detector; EDFA: Erbium-doped fibre amplifier; DFB: distributed feedback laser;

IM: intensity modulator; PS: polarization scrambler. 
It is clear that the real amplification of each pulse in the sequence by the EDFA is different and may even differ from sequence to sequence in the Colour Simplex. Figure 3 shows the monitored power for two different Colour Simplex codewords (sequences 8 and 192 for $L=255$ ). This implies that the power level of each pulse of each sequence has to be monitored during the measurement process. This way, in order to retrieve an undistorted BGS, pump power fluctuations have to be corrected, and therefore, each "1" of the original S coding matrix has actually to be replaced by the weighted pulse power monitored by detector 2 .
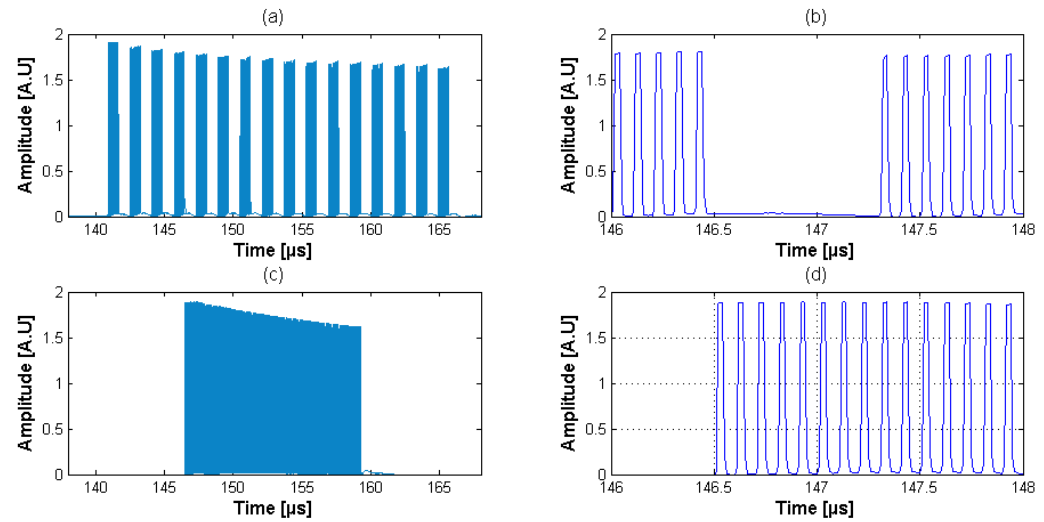

Figure 3: Power measurement of (a) sequence 8 and (c) sequence 192 for $L=255$,

(b) and (d) are enlarged views of (a) and (c), respectively.

\section{MEASUREMENTS AND RESULTS}

In order to validate the concept, a distributed measurement over a $100 \mathrm{~km}$ loop configuration has been performed along a standard G.652 fibre. The spatial resolution was set to 2 meter, in a RZ format. The zero segment duration was set to $68 \mathrm{~ns}$, leaving enough time for the acoustic wave to decay ${ }^{10}$. The code length was set to $L=255$; each coded temporal trace being averaged 1000 times. The 255 distinct frequencies were equally spaced by $1 \mathrm{MHz}$, between $10.754 \mathrm{GHz}$ and $11.008 \mathrm{GHz}$. The pump and probe powers were set below $15 \mathrm{dBm}$ and $-10 \mathrm{dBm}$ respectively, in order to avoid pump depletion and distortion of the amplified probe signal.

Figures 4 and 5 show the decoded BGS and the estimated Brillouin frequency shifts (BFS), respectively, both picked up in the final fibre section. No excessive distortion can be observed on the BGS as a result of the power rescaling of the pump pulses required during the decoding process. The SNR of the decoded traces at the peak Brillouin gain is higher than $5 \mathrm{~dB}$ at the end of the sensing fibre, which means that the Brillouin frequency shift can be perfectly resolved at this distance $^{11}$, with an estimated accuracy better than $\pm 2 \mathrm{MHz}$. Comparing the intensity noise on the temporal traces with the absolute intensity noise calculated from the reconstructed spectral distributions after decoding - such as that shown in Figure 4 - gives an experimental coding gain of about $9 \mathrm{~dB}$, which is in full agreement with the theoretical gain $(9.02 \mathrm{~dB})$.

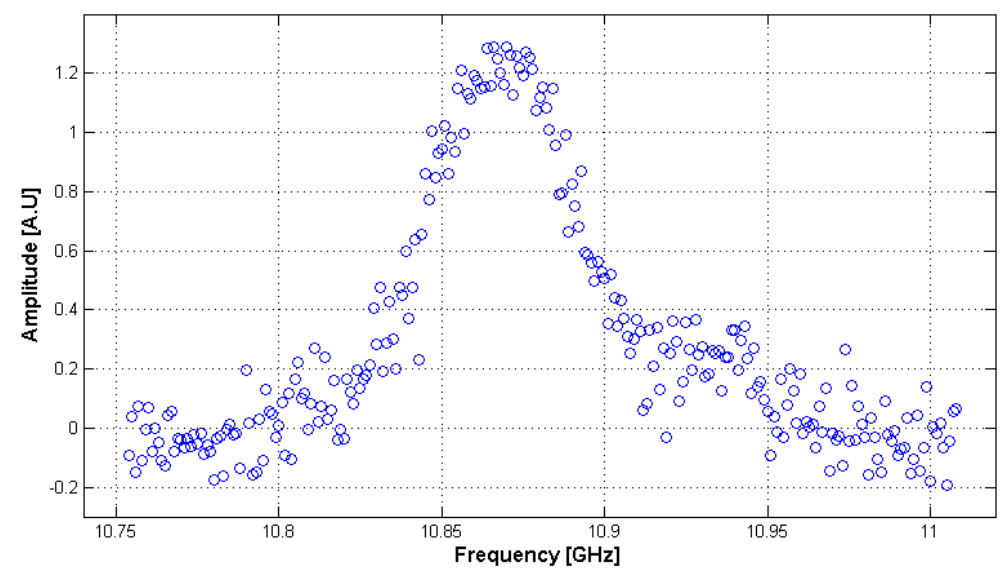

Figure 4: Brillouin gain spectrum (BGS) at a distance of $50 \mathrm{~km}$, retrieved after the decoding process. 


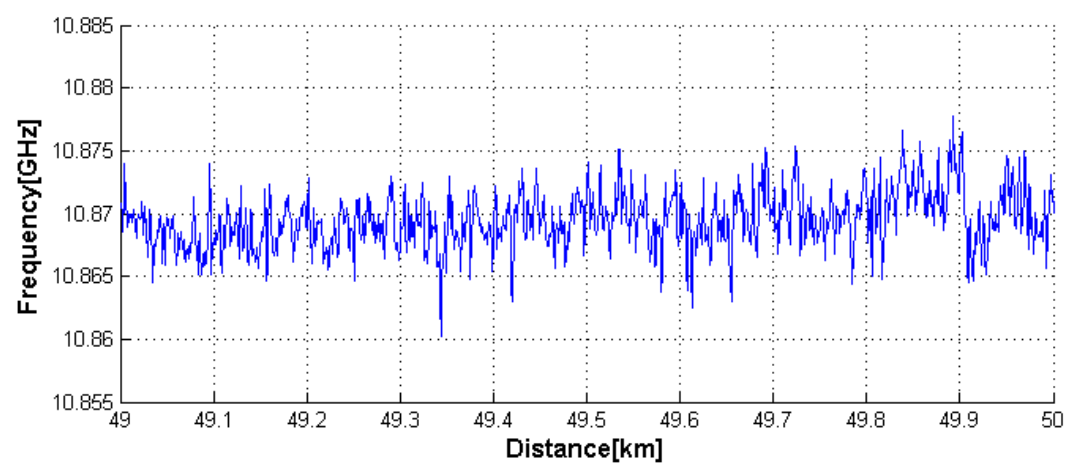

Figure 5: Distribution of Brillouin frequency shift (BFS) along the final kilometre of the sensing fibre.

\section{CONCLUSION}

In conclusion, we have presented a new coding method, designated as Colour Simplex, which can be easily implemented in BOTDA sensors. It shows the advantage of simplicity in terms of number of sequences, since this number matches the number of distinct frequencies used for scanning the BGS. The DDS is a crucial element since, to the best of our knowledge, it is the only microwave generator able to generate short pulses showing random optical frequencies with a very short switching time. The theoretical coding gain was successfully validated by the measurements. This code takes full advantage of the particular conditions to be met for a distributed BGS measurement, namely the double temporal and frequency scanning. This leads to an optimization of the coding in terms of SNR and acquisition time.

\section{ACKNOWLEDGMENTS}

The authors would like to acknowledge the company OMNISENS (Switzerland) and the Swiss Commission for Technology and Innovation (Project 13122.1) for their support.

\section{REFERENCES}

[1] Horiguchi, T., and Tateda, M., "BOTDA—non-destructive measurement of single-mode optical fibre attenuation characteristics using Brillouin interaction: theory”, J. Lightwave Technol. 7, 1170-6 (1989).

[2] Horiguchi, T., Kurashima, T., and Tateda, M., "A technique to measure distributed strain in optical fibres", IEEE Photon. Technol. Lett. 2, 352 (1990).

[3] Fellay, A., Thévenaz, L., Facchini, M., Niklès, M. and Robert, P. "Distributed sensing using stimulated Brillouin scattering : towards ultimate resolution", in 12th International Conference on Optical Fibre Sensors. Technical Digest., 16, 324-327 (1997).

[4] Thévenaz, L., Foaleng, M. F. and Lin, J., "Impact of pump depletion on the determination of the Brillouin gain frequency in distributed fibre sensors", Proc. SPIE 7753, 775322 (2011).

[5] Foaleng, S. M. and Thévenaz, L., "Impact of Raman scattering and modulation instability on the performances of Brillouin sensors", Proc. SPIE 7753, 77539V-1 (2011).

[6] Soto, M. A., Bolognini, G., Di Pasquale, F. and Thévenaz, L., "50 km Simplex-coded BOTDA sensor for distributed strain and temperature measurements with $1 \mathrm{~m}$ spatial resolution", Optics Letters 35(2), 259-261 (2010).

[7] Soto, M. A., Bolognini, G., Di Pasquale, F. and Thévenaz, L., "Long-range Brillouin optical time-domain analysis sensor employing pulse coding techniques", Meas. Sci. Technol. 21, 094024 (2010).

[8] Soto, M. A., Le Floch, S. and Thévenaz, L., "Bipolar pulse coding enhanced performance in Brillouin distributed optical fibre sensors", OFS 2012, 22nd Int. Conf. on Optical Fibre Sensors, Beijing, China (2012).

[9] Le Floch, S., Sauser, F., Soto, M. A. and Thévenaz, L., "Time/frequency coding for Brillouin distributed sensors", OFS 2012, 22nd Int. Conf. on Optical Fibre Sensors, Beijing, China (2012).

[10] Soto, M. A., Bolognini, G. and Di Pasquale, F., "Analysis of pulse modulation format in coded BOTDA sensors", Optics Express 18(14), 14878-14892 (2010).

[11] Thévenaz, L., and Soto, M. A., "Rating the performance of a Brillouin distributed fibre sensor", OFS 2012, 22nd Int. Conf. on Optical Fibre Sensors, Beijing, China (2012). 